\title{
Experience with Memory Management in Open Linda Systems
}

\author{
Ronaldo Menezes \\ Florida Institute of Technology \\ Department of Computer Sciences \\ 150 West University Blvd \\ Melbourne, FL 32901 \\ rmenezes@cs.fit.edu
}

\begin{abstract}
Coordination systems, in particular Linda, have established themselves as important tools for developing applications for open systems such as the Internet.

This paper shows how to tackle a forgotten, yet crucial problem in open coordination systems: memory management. As with any system which intends to be of wide use, coordination systems have to address the problems of memory exhaustion since memory is a finite resource. This paper first explores the separation between coordination and computation in order to make it clear that the problem of memory exhaustion in coordination systems cannot be solved using garbage collection schemes implemented at the computation language - a garbage collection scheme must exist in the coordination environment as well.

As Linda is arguably the most successful coordination system, this paper will focus on the Linda-like family of systems. By showing how the problem can be tackled in Linda it is expected that a solution for other coordination system could be implemented by adapting the method described.
\end{abstract}

\section{Keywords}

coordination systems, garbage collection, open systems, Linda

\section{INTRODUCTION}

Linda has surely caused an impact in the computer science community. Since its proposal by Gelernter [11] the basic idea of Linda has evolved to a point of even attracting the attention of the industry. Recently two major computer companies have released commercial packages based on Linda: Sun Microsystems with JavaSpaces [29] and IBM with T Spaces [16].

The path to achieve such a recognition was not easy and it was necessary nearly 15 years of research to convince the world of Linda's abilities. In its evolution, Linda has

Permission to make digital or hard copies of all or part of this work for personal or classroom use is granted without fee provided that copies are not made or distributed for profit or commercial advantage and that copies bear this notice and the full citation on the first page. To copy otherwise, to republish, to post on servers or to redistribute to lists, requires prior specific permission and/or a fee.

SAC 2001, Las Vegas, NV

Copyright 2001 ACM 1-58113-324-3/01/02 ..\$5.00 changed from a model aimed at parallel computing to a model aimed at distributed computing, in particular, interesting for open distributed computing.

The first implementations of Linda were confined to closed (controlled) environments $[27,26]$. In the closed case most of the problems are of little concern as they can be dealt with at compile-time. However the same is not true in open implementations of Linda. Any existing problem handled at compile-time in closed systems becomes a concern in the open case as the solutions have to be implemented at runtime involving dynamic reconfiguration of data structures and more control over race-conditions.

Additionally, problems in closed systems are confined to the boundaries of a single computation language, which hides the idea that coordination and computation are orthogonal concepts because the computation language defines a boundary to coordination giving the impression that the computation language itself deals with coordination. In open systems the situation is rather different. Open applications can be implemented over different languages, different machines, different operating systems, that is, a complete heterogeneous environment. This heterogeneity stops computation languages from being able to deal with coordination problems since the problems break the boundaries of computation languages. Hence, coordination problems need to be tackled at the coordination level - within the coordination environment.

The aim of this paper is twofold:

1. Develop a way of keeping enough information about Linda objects within the kernel so that optimizations can be implemented based on this information.

2. Describe how the information maintained can be used to tackle the memory management problem in Linda.

Garbage collection of coordination objects cannot be performed at the computation language level. Instead, a garbage collection scheme has to be implemented so as to collect solely coordination objects. With the introduction of the multiple tuple spaces concept in Linda [12], processes became able to create tuple spaces via a primitive provided. However, in open implementations of Linda, these tuple spaces remain 'forever' in the system once they are created. This paper argues that the implementation of a garbage collection system within Linda is the only possible solution to avoid memory exhaustion of happening when large applications are implemented in open Linda systems. 
This paper is divided as follow. In Section 2 the background work is discussed. First the orthogonality argument of Gelernter and Carriero [13] is explained as this is an essential concept for the understanding of why the memory resources at the coordination level cannot be managed by computational languages. Then a brief introduction to the Linda models is given followed by a discussion on open implementations of this model. Section 3 explains the memory exhaustion problem and why it can only be solved if more information becomes available at the kernel level. Section 4 shows how information about accessibility of tuple spaces by processes can be gathered in a distributed graph structure. Section 5 shows how one should go about implementing garbage collection using the distributed graph present at the kernel level. Section 6 presents some experimental results extracted from the implementation of the garbage collection described. Finally, Section 7 presents the conclusion of this paper.

\section{BACKGROUND}

\subsection{Computation vs. Coordination}

Although for some the orthogonality between computation and coordination may be very clear, only recently this concept has been formally proposed. After the introduction of the Linda model [11] and its subsequent extension to the multiple tuple space model, the idea of Linda as a model exclusive for parallel computing was replaced by the idea of having Linda as a model for distributed computing. However, Linda does not represent a distributed system by itself, its implementation has to be embedded in languages like $\mathrm{C}$ [28], Prolog [31], ISETL [8], and others.

Gelernter and Carriero observing this characteristic argued that Linda does not deal with computation but only with coordination aspects of a distributed system - Linda is a coordination model [13]. The idea can be easily observed with an example: if two processes are each generating a number that will be added by a third process, coordination models are not interested in how the addition is carried out but in how the two numbers are obtained before the operation - coordination is the process of managing dependencies between distributed activities. Soon after the term coordination become accepted by the computer science community, other existing models, like Actors [1] and CHAM [2] were also classified as coordination models since they, like Linda, are not concerned with computation.

In reality, Gelernter and Carriero, did not create the term coordination, they have only described how the coordination idea introduced by Malone and Crowston [19], could be applied on the design of distributed computer systems. They have described a distributed language in terms of coordination and computation and have shown that a coordination model (or language) when put together with a computation language form a complete distributed language.

\subsection{The Linda Coordination Model}

The Linda coordination model $[11,12]$ is based on the concept of generative communication via distributed associative shared memories. The shared memories are called tuple spaces and behave like bags (unordered multi-sets) of tuples. There is no direct communication between processes; all communication is done via tuple spaces. The model provides the processes with primitives to access the tuple spaces: storing and retrieving tuples, creating tuple spaces, and spawning other processes.

Tuples in Linda are ordered lists of valued-objects (actuals). The generative communication model is based on associative matching implemented on the primitives. The matching implemented in Linda is based on templates which are no different from tuples except for being able to have non-valued objects (formals) represented by ?type. For instance the template [?int, "Hello"] matches the tuple [1, "Hello"].

The primitives provided are $^{1}$ : out (stores a tuple in a tuple space), in (removes a tuple from a tuple space matching the template provided), rd (gets a copy a tuple from a tuple space matching the template provided), collect (moves all tuples matching the template from a tuple space to another), copy (copies all tuples matching the template from a tuple space to another) and eval (spawns a new process). By using these primitives, Linda unifies the concepts of process creation, communication and synchronization since they are all implemented as tuple space operations.

There are several variants of the Linda model (called nowadays the Linda family of models) among which deserve mentioning: Melinda [15], Bauhaus [5], PageSpace [6] and Bonita [25]. Despite their differences all the models would suffer from the same problem should they be implemented in open environments: memory exhaustion.

\subsection{Open Implementations}

One might wonder why the memory exhaustion problem has not been felt to a great extent so far. The answer is simple; only recently open implementations of Linda started to appear and even the ones already available have not yet been used on the implementation of large applications. However the increase of commercial open implementations of Linda - like JavaSpaces and T Spaces — will in a short period of time make Linda a viable commercial product for the developing of open applications. An example of this phenomenon can be observed with Sun Microsystems' Jini [30] which includes JavaSpaces as one of its components. Yet, Jini is not "the" perfect example because JavaSpaces (coordination) is confined to a single computation language (Java).

In open implementations of coordination systems problems that were previously solved at compile-time, are now required to be solved separately, by a run-time sub-system. To make matters worse, even vital information necessary for the implementation of these sub-systems is no longer available. For instance, in a closed system, it is possible for the kernel to built at compile-time a structure containing the information of which processes are likely to access which tuple spaces. With this information in hand, decisions (optimizations or garbage collection for instance) can be made without interfering in the execution of the system.

Generally speaking one can say that due to the existence of enough information in closed systems the solutions for possible problems are easier. In other words, the degree of difficulty of problems in open systems is higher than the same problems for closed systems. As the majority of problems in closed systems can be dealt with at compile-time, the solutions can use stable (passive) structures containing the information. In open systems not only the information has

\footnotetext{
${ }^{1}$ collect and copy are not standard primitives but the concept of bulk primitives is well accepted for them to be included here.
} 
to be maintained at run-time but also the solutions have to be careful to use the information available at the right time when the information is coherent with the state of the system.

\section{MEMORY EXHAUSTION}

Memory management hunts every large application developer. As computers becomes cheaper, systems tend to be larger and use more of the memory resources. However the bottom line is that memory being a finite resource, its utilization is (and always will be) a concern.

As mentioned before, the problem has not yet been observed in Linda systems. There are a number of reasons for this:

- as the first commercial implementations of Linda were closed [27, 26], compile-time optimizations implemented at the kernel level were used to manage memory usage;

- in closed system the orthogonality of coordination and computation is not observed since both concepts are mingled into one distributed/parallel language;

- large, memory demanding applications have not been implemented using closed Linda implementations because most of them demand open systems;

- open implementations of Linda are largely research prototypes where only small applications (test programs) are implemented making memory management not and issue;

- only recently companies like Sun [29] and IBM [16] started to explore the possibilities of open Linda-like implementations in the Internet context but their implementation are still to be used in the development of large open applications.

Still, the applicability of coordination systems to the Web and the benefits they can bring to the Internet are well known and accepted. Therefore, it is correct to think that it will not be long until the problem of memory management start to appear. Palliative solutions can be implemented but this paper looks ahead and identify the core of the problem: lack of information within the system.

\section{INFORMATION GATHERING}

Open implementations of the Linda model have to find means of building up information about processes, tuple spaces and tuples in the kernel. This can be done by using for instance pre-processing, using active agents for gathering information at run-time [18], or gathering the information using the messages in transit within the system [22].

The use of pre-processing can, in some cases, build an initial amount of information that allows some optimization, however, as pre-processing is normally done from a single process point of view it does not help in the solution of global problems like the problem of memory management. The solution based on active agents although very elegant might add undesirable overhead to the system. This paper explores how to solve the problem of memory management based on the idea of gathering information using the messages in transit between the Linda processes and the Linda kernel. It is trivial to see that most of the information necessary to build a structure that could be made available to sub-system processes (such as garbage collection) is already in transit in the Linda case. The problem is that this information is volatile - because it is not stored, it is lost.

In order to add some persistence to the information in transit within the system, a distributed graph ${ }^{2}$ with the information of which processes access which tuple spaces is built in the Linda kernel.

\subsection{Consideration of Tuples}

Before discussing how the graph is build it is worth clarifying why tuples will not be considered. One could naively think that as tuples are the basic elements being stored and retrieved in Linda, any memory management should be done at the tuple level. The reason for not doing this is that tuples in Linda are not uniquely identified. A tuple is just an ordered list of values and processes do not keep references to them. Yet, processes do keep references to tuple spaces (bag of tuples) as their name have to be of a process knowledge if this intends to stored/retrieve tuple in/from them.

One solution that is worth mentioning is the idea of leases used in JavaSpaces. The basic idea is that resources can be allocated for a fixed period of time. For instance, a tuple is created with a "expiration date". Freeman et Al. [10] argue that leases are ideal for unreliable distributed applications where processes can fail before resources are explicitly freed. While lease can be used in certain cases where the behavior of the application is known, it cannot be used when the behavior is unpredictable. In asynchronous system a process cannot predict for how long a tuple will be required in the system nor can the process delete any tuple explicit due to the same reason. This paper argues that not even the kernel can free resources at the tuple level because tuples are accessed associatively - there is no handle to a tuple. Leases work in JavaSpaces because tuples are confined to a single language and are represented as Java objects.

\subsection{Using the Messages in Transit}

This paper therefore concentrates in having a graph with information about tuple spaces and processes only. The construction of the distributed graph is based on two concepts that need to be introduced within the kernel, so that the information in transit does not get lost.

Process Registration/Check-out: The kernel should be aware of all processes willing to coordinate using Linda primitives. Process registration consists of having each process communicating with the kernel so that this can assign them with a unique name. From the point of registration onwards all processes will use their internal names in all communication with the kernel.

The dual of the process registration is the process check-out where the processes informs the Linda kernel that it does not intend to use Linda facilities any longer.

Tuple Monitoring: Tuple monitoring is the idea of having the Linda kernel analyzing all relevant information in transit. It is called tuple monitoring because the basic information in transit in Linda are tuples. Since

\footnotetext{
${ }^{2}$ Despite being called a graph, the name stands only for the pictorial representation of the data structure containing the information itself.
} 
processes can be identified by their names, the tuple monitoring is effective in extracting information stored within tuples.

With the implementation of the concepts above, Linda is being given more power as it becomes aware of what is happening in all communications. As processes and tuple spaces are uniquely identified the so-called distributed graph can be built to hold information about the system.

Although the implementation of the concepts above is crucial for the solution of the memory management problem they are not just a commodity that is being implemented only to satisfy the garbage collection scheme. The graph is building up information on the kernel that can be made available to other optimization run-time systems.

\subsection{Creating Distributed Graphs}

Clearly, with the information available in the kernel after the introduction of the concepts described in Section 4.2 a data structure can be built. As said before the intention is to build a data structure (pictorially seen as a distributed graph) with the information of tuple spaces usage by processes. Due to the distributed characteristic of this graph some rules have to be clarified:

- all existing tuple spaces and processes are represented in the graph;

- there is only one representation for a process and a tuple space across all distributed components of the graph;

- in order to observe the distributed components as part of a whole (connected) graph the representation of the Universal Tuple Space $(U T S)^{3}$ is repeated in all components to serve as the connection among all components;

- processes hold references to tuple spaces and tuple spaces might store references to other tuple spaces but processes do not hold references of other processes references are represented as edges in the graph;

- for the purpose of garbage collection algorithm, edges linking a processes and a tuple space are undirected whereas edges linking two tuple spaces are directed, outgoing from the tuple space that contains the reference;

- directed edges are weighted with the number of references stored in the outgoing tuple space;

- both directed and undirected edges are never duplicated - given two nodes there is a maximum of one undirected edge between them or two directed edges (one in each direction).

Based on what has been described above, Figure 1 could represent the situation of the data structure in one of the locations (one component of the graph). The figure represents a scenario where four processes are executing and because they all are aware of the existence of UTS there is an edge (undirected) linking each one of them to UTS. The figure is

\footnotetext{
${ }^{3}$ Its is generally the case that Linda systems have a medium of communication which is known to all processes.
}

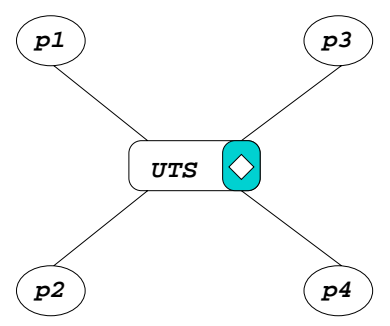

Figure 1: Simple graph situation.

equivalent to have the following adjacency matrix $\{\{p 1\{U T S$ \}$\},\{p 2\{U T S\}\},\{p 3\{U T S\}\},\{p 4\{U T S\}\},\{U T S\{p 1, p 2, p 3, p 4$ \}\}\}.

There are several ways to get to a particular graph configuration. The one shown in Figure 1 may have got to this configuration by having the four processes starting soon after the kernel was initialized. During the initialization the node UTS is created. Then when a processes registers with the kernel a name is attributed to the processes, a node is created representing it and the necessary edges are added, in this case only the edge linking them to UTS.

For a better understanding of the garbage collection algorithm used, the nodes of tuple spaces in the graph contains also an extra field which represents the number of references from other objects (tuple spaces and processes) to this tuple space. In the UTS case it is set as $\diamond$ to represent that UTS has infinite number of references — by default, all processes know about UTS.

Undirected edges are not weighted. A process (an active object) once it knows about a tuple space it can generate as many references (tuple space handles) as it likes. Therefore the undirected edges can be understood as having infinite weight.

A more complex graph is shown in Figure 2. Again there are numerous ways of getting to the situation depicted. Before explaining in more details how the structure is built it is important to be convinced of the importance of keeping this information in the kernel. The graph in Figure 2 holds important information that is normally not available in Linda systems. For instance it is easy to see that tuple space $t s 7$ is not being used by any process and that its handle is not available within any other tuple space. Basically, this tuple space is garbage and could be garbage collected - the kernel now maintains information that was not available before. Observe that although the system being considered is fully open, because tuple spaces are accessed by their names it is impossible to any process to get the handle of $t s 7$. As another example suppose that both processes $p 4$ and $p 5$ terminate. Should this happen, their representations will be removed from the graph causing tuple spaces $t s 3$, $t s 6$ and $t s 9$ to be unreachable to all other processes and consequently be considered garbage.

Consider yet just another example as shown in Figure 3. This scenario shows how the distributed graph can help on the implementation of mobility in Linda. There is a big interest on mobile computing today mainly due to the availability of the Web as a distributed network for implementation of systems that span the globe. Static techniques adapted from Local Area Networks (LANs) like RMI/RPC and Corba, although successful in fairly large Wide Area 


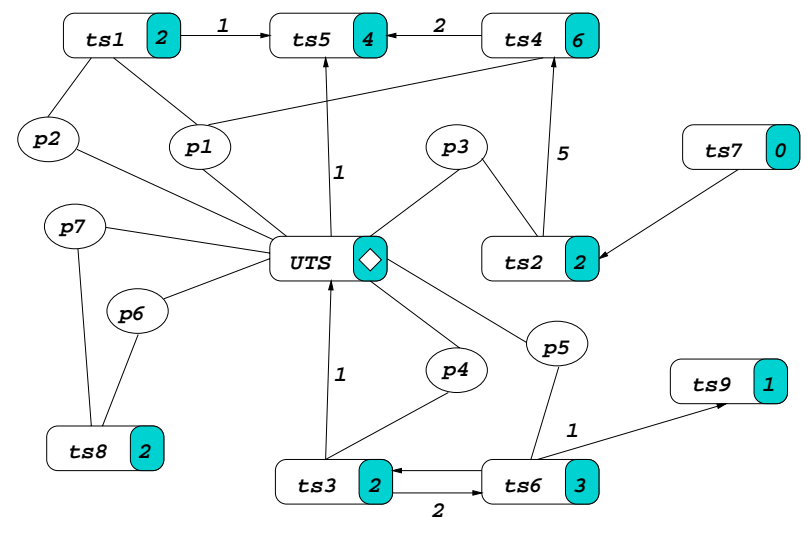

Figure 2: A more complex graph situation.

Networks (WANs) are not well adapted to the Web context [4]. If mobility is to be implemented in Linda the information provided by the distributed graph considered here is of utterly importance. Figure 3 shows a simple case where process $p 5$ is the sole process accessing the tuple space $t s 1$. Since $p 5$ is in a different location this might say to a mobile system that $t s 1$ should migrate to location B or alternatively process $p 5$ should migrate to location A (given that this process is also not accessing other tuple spaces in location B).

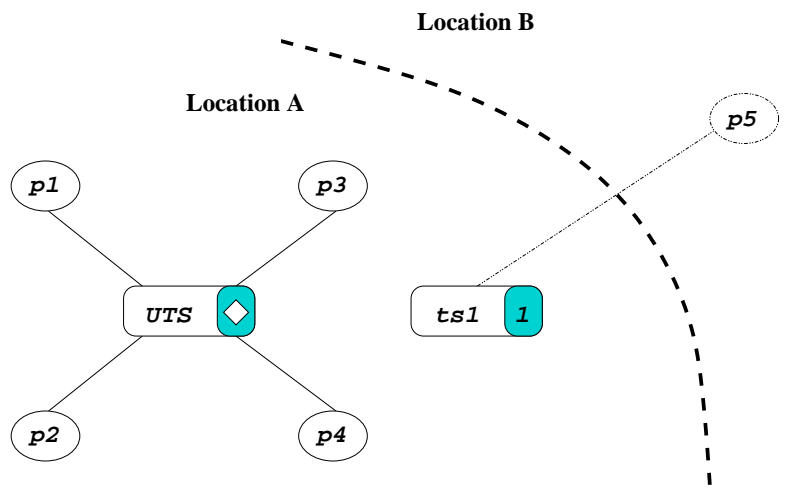

Figure 3: A simple case of distributed graphs.

The information put together in the graphs depicted so far is made available by the two concepts described in Section 4.2. First process registration allows the kernel to create a node for the process in the graph as well as identify all messages related to this process from the moment of registration onwards. During the registration some links can also be created to existing tuple spaces if the process is spawned receiving handles as parameters.

Tuple monitoring is used to keep the graph updated. It behaves as an independent process that basically listens to the messages being transmitted between the Linda kernel and processes and vice-versa. Although all messages in transit are potentially important, it is possible for messages to be identified so that only three types of messages are considered:

- messages requesting process registration;
- messages storing or retrieving tuples containing handles;

- messages requesting process check-out;

Although process registration and check-out is dealt with separately, it is up to the tuple monitoring to identify the message as a registration or a check-out request. The messages are marked when they are mounted at the Linda process side. This flagging does not impose big overhead to the system as it occurs when the tuple is packed to be sent to the server.

The tuple monitoring update the graph in several ways. For every message storing a tuple that contains a handle (via out, copy or collect) a directed edge has to be created in the graph to represent a dependency between the tuple spaces involved - if a tuple space $t s x$ contains a handle of $t s y$, any process with access to $t s x$ has potential access to $t s y$ by retrieving its handle. If a directed edge already exist linking the two tuple spaces, its weight is modified accordingly since the weight represents the number of handles stored in the tuple space. The other messages monitored are the ones retrieving handles from a tuple spaces (in, rd, copy and collect) ${ }^{4}$. If a handle is removed from a tuple space the weight has to be updated to reflect the change, and if the weight of the directed edge gets to zero the edge is removed altogether. The retrieval of handles can grant a process access to a tuple space, therefore not only the necessary directed edges are updated but, if necessary, an undirected edge between th process and the tuple space has to be created.

\subsection{Avoiding Race-Conditions}

The operations that update the graph must be done with care in order to avoid race conditions. Observe for instance a case with primitive $i n$. Suppose that in the scenario depicted in Figure 2 process $p 5$ removes the tuple containing the handle of $t s 9$ from $t s 6$. If a garbage collection scheme is using the graph to decide on what is garbage and what is not, a race condition might occur between the garbage collector and the update of the graph. When process $p 5$ removes the handle it automatically gains access to $t s 9$, however if the order of update of links is not observed tuple space $t s 9$ can be considered garbage. If the edge linking $t s 6$ to $t s 9$ is removed before one linking $p 5$ and $t s 9$ is created.

One of the characteristics of the update method used is that garbage is only created by process termination. Removal of directed edges does not, in any circumstance, generate garbage. The edges in the graph represent knowledge of a particular tuple space, if one is removed due to some process operation another will be created (or modified in terms of weight). References to a particular tuple space are never lost due to execution of Linda primitives.

On the other hand process termination is an operation that can generate garbage as references may be lost. Race conditions between garbage collection and graph update is a well know problem in distributed system and has been solved using various approaches developed through the years $[14,3,24]$. The adaptation of these models for the Linda case would avoid the race-condition. Yet, this paper uses a different approach and instead of adapting one of these

\footnotetext{
${ }^{4}$ Bulk primitives, collect and copy, both store and retrieve tuples from tuple spaces.
} 
(more complex) algorithms, uses a simple one and avoids the race conditions by guaranteeing that at any time the graph is consistent with the system situation.

Douglas et Al. [9] have described the out-ordering problem showing that this is a problem that must be solved in any Linda-like coordination system. The problem consists of guaranteeing that the order of execution of a sequence of outs to a tuple space $t s x$ is the same order that this tuples appear within $t s x$. The solution for avoiding race conditions in the graph can be seen as an extension of this problem where some ordering is also imposed to the checkout message sent by the Linda processes. Race conditions are removed if the system guarantees that after the checkout message arrives in the kernel, no other message from the same process can arrive. The experiments shown in Section 6 show that this solution does not impose a great overhead to the system.

The argument that can be used to show that little overhead is added by this extra ordering is based on the fact that only the primitive out needs to be considered in the ordering. Other primitives always return something to the process therefore ordering already exists as part of the semantics of the primitive. For instance, suppose that a primitive $i n$ is the last primitive executed before the process checks-out. As in returns a tuple the check-out operation immediately following would not even be executed at the process side until the primitive in is completed, thus guaranteeing the ordering of execution. The primitive out is the only one that does not return a value and consequently the only one that needs to be considered in the ordering with termination (check-out).

\section{GARBAGE COLLECTION}

Garbage collection in Linda is implemented at the kernel level as this is the only way to guarantee that the scheme would not be restricted to boundaries of computation languages. The scheme implemented aims at finding garbage tuple spaces but some consideration is given to what would be required to garbage collect Linda processes as well.

\subsection{Finding Garbage Tuple Spaces}

Given that the information now available in the kernel the implementation of a garbage collection scheme for finding tuple spaces which are no longer necessary in the system is simple. The most popular garbage collection algorithms available are variations of one of the two basic methods: Reference Counting[7] and Mark-and Sweep[20].

The scheme implemented in Linda is based on a two-phase garbage collection. The first phase uses reference counting to find tuple spaces that are not required within the system. Basically any tuple space that has its counter field (number of processes with knowledge of this tuple space) zero is wasting memory space and can be removed. Figure 4 shows a scenario where two tuple spaces, $t s 7$ and $t s 13$, can be removed by the garbage collector since their counters are zero.

However, reference counting methods do not always find all garbage; they do not work well in cyclic structures since cyclic garbage are not identified. Yet, reference counting is cheap to be implemented in distributed environments [17] therefore justifying the option of implementing a scheme in two phases. The phases are implemented in such a way that the reference counting runs more frequently and ideally

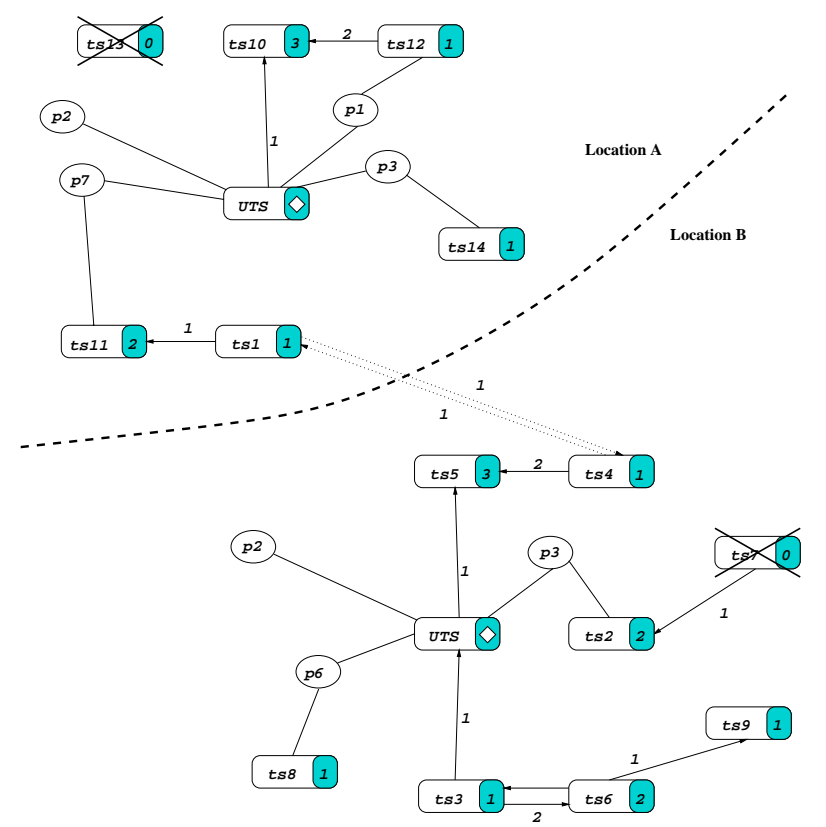

Figure 4: Reference counting identifies two tuple spaces as garbage.

collects most of the garbage.

A second phase is nevertheless important so that cyclic garbage is collected. Mark-and-sweep is able to collect cycles because it does not decide on the need of an object based on its counter. Instead, mark-and-sweep traverses the graph marking everything that can be reached from special nodes called roots. In Linda UTS can be nominated as root because it represents the boundary to the outside world (non-linda environment). Processes, being active objects, could also act as roots in the search but because they are all linked to UTS this is not necessary - if a node is reached from a process this node is also reached from UTS.

The mark-and-sweep has been implemented using a concept based on sets. In the marking step, any node reached is included in a set that represent the nodes alive. In the end of the marking the nodes left outside the set are considered garbage and can be collected by the sweep step. By performing a marking in the scenario depicted in Figure 4 more garbage can be found, as shown in Figure 5 .

It should be clear why the tuple spaces identified as garbage (crossed) in this phase are indeed garbage. Take for instance the case of tuple spaces $t s 3, t s 6$ and $t s 9$. Although $t s 3$ contains a reference to UTS, the traversal that started from the UTS cannot reach any of these tuple spaces - the search respects the direction of the edges. Also there are no processes accessing them, in fact if there were, the search from UTS would have reached the tuple spaces.

The mark-and-sweep phase itself can be divided in two stages: a local and a global mark-and-sweep. Given that the representations of tuple spaces in the graph contain some information about the location of the objects with access to a particular tuple space, local and global mark-and-sweep can be used. In the scenario shown in Figure 5, $t s 3, t s 6$ and $t s 9$ would be collected by a local mark-and-sweep since none of them have links to other locations whereas $t s 1$ and 


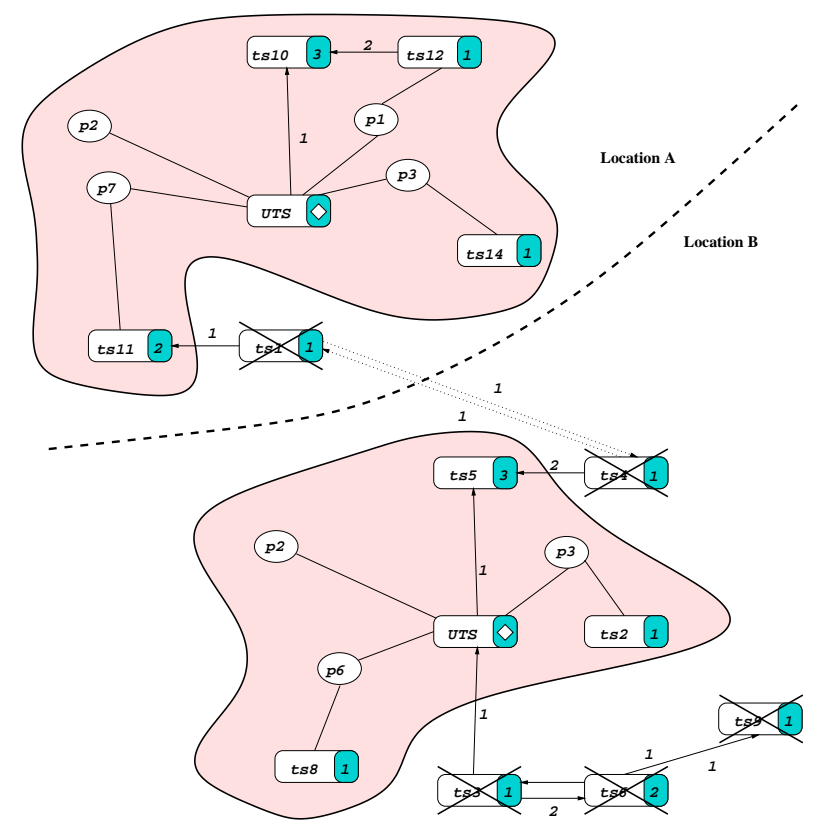

Figure 5: Marking identifies five tuple spaces as garbage and the sweep step collects them.

ts 4 would be collected by a global mark-and-sweep, as this would have the view of the whole distributed graph.

\subsection{Considering Active Processes}

Extending the concept of memory management to resource management, can the graph help on garbage collecting processes which although running are only wasting processor time? In other words, is it possible to have a process in Linda that although active cannot communicate with other processes and therefore whatever it is doing it is just waste of time? The way Linda is usually implemented, this does not happen because as all processes are linked to UTS they can always side-effect other processes. Surely if this condition is relaxed by having not all processes aware of the existence of UTS the graph would provide information so that garbage collection of active processes can be done.

It should be noticed that the concept of garbage collection of processes here does not include garbage collection of deadlocked processes which are for instance blocked (via in or $r d$ ) waiting for a tuple. This should not be dealt with by a garbage collection scheme, instead the semantics of the primitive should foresee this situation and deal with it.

UTS as a tuple space has handles that can be passed to other processes via tuple spaces. This would mean to say that the assumption that all processes know by default about UTS can be considered too general and unnecessary.

In order to garbage collect processes the scheme described in Section 5.1 can be used but processes have now to be considered as roots of the graph as not all of them are linked to UTS. As active objects, even if they are not linked to UTS they can get access to UTS by retrieving its handle. This has to be considered in the mark-and-sweep phase which need to search the graph starting from the processes nodes as well as UTS. However UTS remains the center of the algorithm. In the end of the search a given node $x$ is garbage if:
1. it is not contained in any set generated during the search; or,

2. it is contained in a set but UTS is not an element of it.

The creation of the sets during the marking is very simple. If the search has started from a node $y$, all nodes reached in this search will be elements of the set $Y$ and they are marked as reached by $y$. Given a set $Y$ and a set $Z$ if $Y \cap Z \neq \emptyset$ then $Y$ and $Z$ have to merge forming a single set.

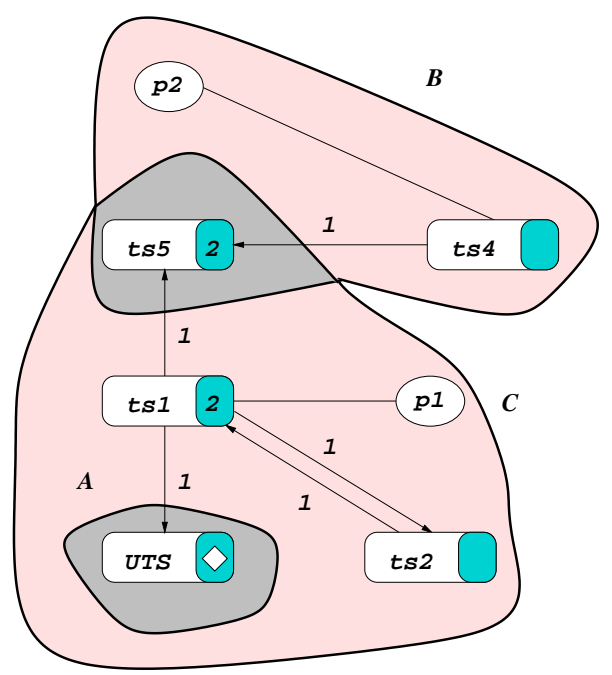

Figure 6: Scenarios showing how sets merge during the marking.

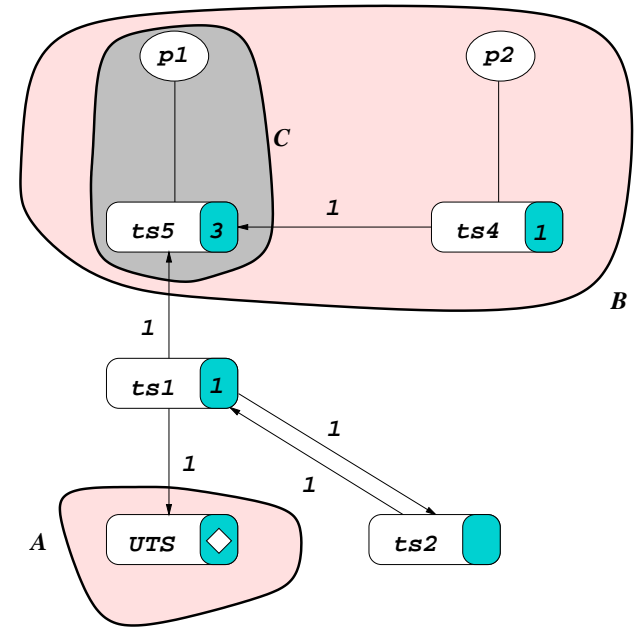

Figure 7: Scenarios showing how sets merge during the marking.

Suppose the two scenarios in Figures 6 and 7 which are now feasible given that not all processes are linked to UTS. Figure 6 shows three sets $A, C$ and $B$ that were generated by the marking phase starting from UTS, $p 1$ and $p 2$ respectively. In this figure none of the objects are garbage because the sets can merge forming one single set which contains UTS 
as one of its elements. Using the property described above, because $B \cap C=\{t s 5\}$, they merge forming one single set, say $D$. Now $D \cap A=\{U T S\}$ means that $A$ and $D$ also merge. In the end of all merge operations a single set given by $\{U T S, p 1, p 2, t s 1, t s 2, t s 4, t s 5\}$ is formed. This set represents the nodes that are not garbage.

Figure 7 shows how the marking phase can find garbage nodes. Similar to the case in Figure 6 sets $B$ and $C$ merge because they have elements in common. However the set resulting of the merge, say $D$, does not have an element in common with $A$, that is, $D \cap A=\emptyset$. Therefore in the end of the marking the following applies:

- ts1 and ts2 are garbage because they do not belong to any set;

- $p 1, p 2, t s 5$ and $t s 4$ are also garbage because UTS is not an element of the set they belong, $D$;

- UTS will be the only node left in the graph.

\subsection{The I/O Side-Effect}

Given the method described so far a last question remains: what happens if a process is not linked to UTS but intends to display some result on the screen? This in fact might be a very common situation where a process $p x$ starts, creates a tuple space $t s x$, spawns another process $p z$ passing the tuple space handle of $t s x$, and terminates. $p z$, in turn, will access information in $t s x$ and display some result. Surely one does not want to garbage collect processes doing I/O even though they are not linked to UTS in anyway.

Menezes and Wood [21] have shown that I/O is a coordination problem and must be addressed within the coordination model. The authors described how can devices and files be abstracted in terms of tuple spaces allowing Linda processes to deal with them using Linda primitives. In the context of garbage collection this can be represented by adding a tuple space that represents $\mathrm{I} / \mathrm{O}$ within the model. If a process has the handle of this $I / O$ tuple space this process is able to do $I / O$ operations or in other words abstract devices and files as tuple spaces.

This slightly modifies the concept of garbage defined in Section 5.2. The modification is due to the existence of a new tuple space which is root of the graph: I/O. A node that is element of a set that does not contain UTS but contains I/O as element is not considered garbage. The practical reason for this is that a node that can reach the $I / O$ tuple space can in the Linda model affect the outside world (non-Linda environment) by doing I/O operations.

Figure 8 shows the case where an $I / O$ tuple space exist. In the scenario shown $p 6$ and $p 7$, although not linked to UTS in anyway are not garbage because the definition of garbage has to consider the reachability of the $I / O$ tuple space in the same way used to UTS - the set formed from a search from either $p 6$ or $p 7$ will include $I / 0$ as an element.

\section{EXPERIMENTAL RESULTS}

The system described in the previous sections was implemented using Java. All the concepts described were implemented in a Linda-like kernel called Ligia [23].

The first experiment, described in Figure 9, aims at showing that garbage collection is in fact avoiding early memory exceptions due to the existence of unnecessary information in the memory.

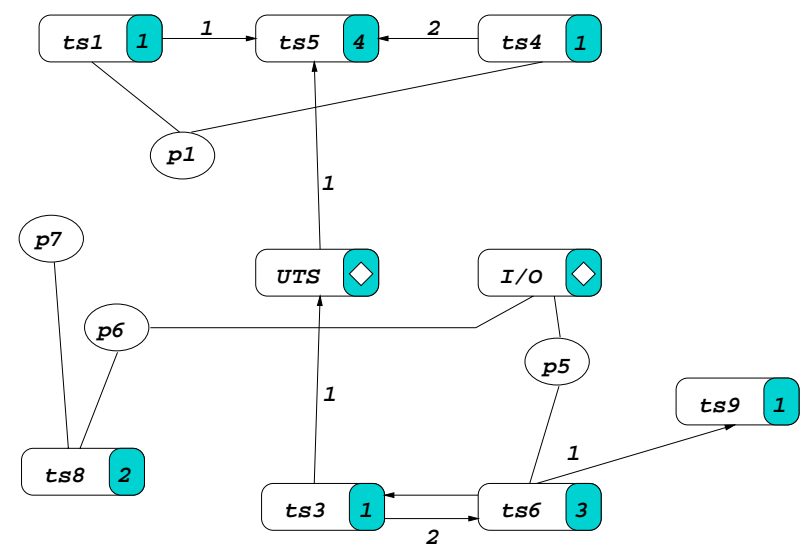

Figure 8: Graph showing the UTS and the I/O tuple space where not all process have their handles.

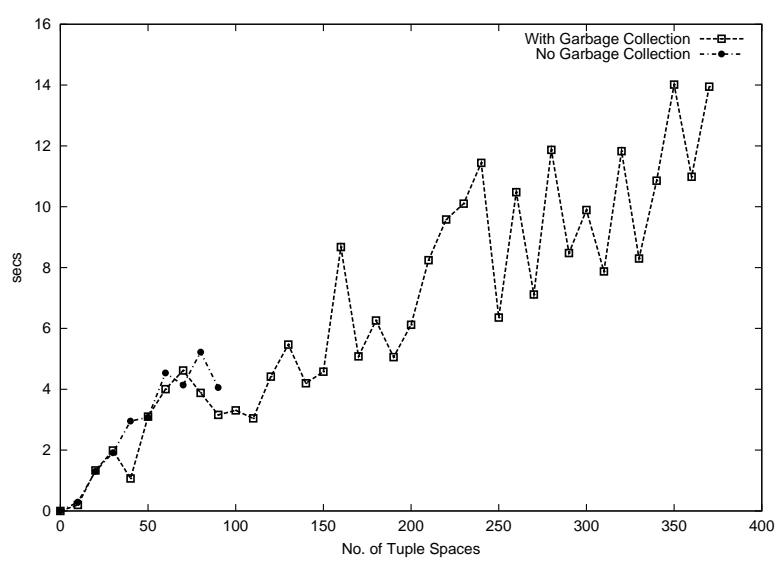

Figure 9: Avoiding early memory exhaustion.

The experiment described was performed using several Linda processes that basically start, create $n$ tuple spaces and terminates. For each new process starting the number of tuple spaces created is incremented by 10 . The system was configured in a manner that memory exhaustion happens when around 450 tuple spaces are stored in memory $(10+20+30+\ldots+90)$. Figure 9 shows that when the garbage collector is not active, the memory exhaustion problem happens when a process is about to create 100 tuple space because there are already 450 tuple spaces stored in memory. When the garbage collector is active the exception does not happen at the same point because the garbage tuple spaces are removed from memory when the processes terminate. With garbage collector active the exception occurs when a process start and tries to create 370 tuple spaces.

Although the capacity of the memory is around 450 tuple spaces the exhaustion happens when 370 are being created due to concurrent nature of the garbage collector. The garbage collector is running concurrently with the execution of Linda processes which means that some garbage may still be in the memory when the exception happened. This could be avoided by implementing an exception handling system which calls the garbage collector and only if this cannot free any memory the exception takes place. However, the system 
used was implemented in Java and the OutOfMemoryError is one of the exception that cannot be handled in Java.

Overhead is one of the biggest concerns when implementing a garbage collection scheme. Figure 10 demonstrates the raw overhead of the garbage collector described previously.

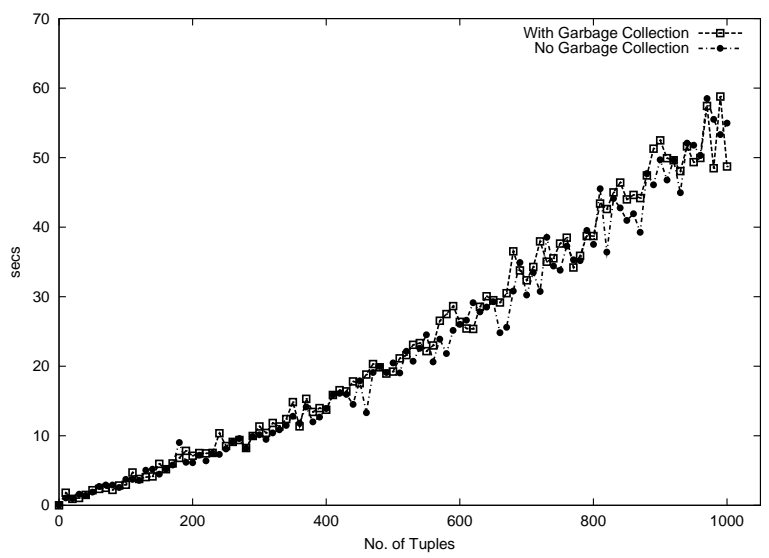

Figure 10: Worst case overhead added by the garbage collector.

The experiment shown in Figure 10 was performed by having a Linda process storing tuples in a single tuple space. The process loops and in each interaction its increases the number of tuple being stored by 10 . Because all tuples are being stored by a single processes into a single tuple space, no garbage is created until the process terminates. Therefore the overhead observed in Figure 10 accounts for the tuple monitoring and the overhead of having the garbage collector running. Process registration/check-out is not considered because it only happens once per process; it adds a startup time which does not have influence on the process total running time.

The average overhead observed in Figure 10 was around $5 \%$ which is bellow what Jones and Lins have argued as acceptable in terms of overhead due to garbage collection [17]. Yet, this is the worst case of the overhead observed because the system is not benefiting from any improvement done by the garbage collector. It is natural to believe that if a system generates an excessive amount of garbage, the execution of the garbage collector can improve the performance of the system by keeping the memory tidy.

Figure 11 shows a scenario which is the opposite of the case in Figure 10. The process loops similarly to the previous case, but instead of storing the tuples within a single tuple space the process spawns another processes which in turn create a tuple space, store the tuples and terminate garbage is created.

Although the results shown in Figure 11 may be surprising, it should be noticed that the situation is the best case analysis for the garbage collection because, as opposed to the case in Figure 10, all tuple spaces created become garbage very quickly. Without garbage collection, the data structure of tuple spaces and tuples become very 'heavy' and operations take longer to complete. When the garbage collector is running and collecting all the garbage, the structure is most of the time 'clean', and operations take less time to complete.

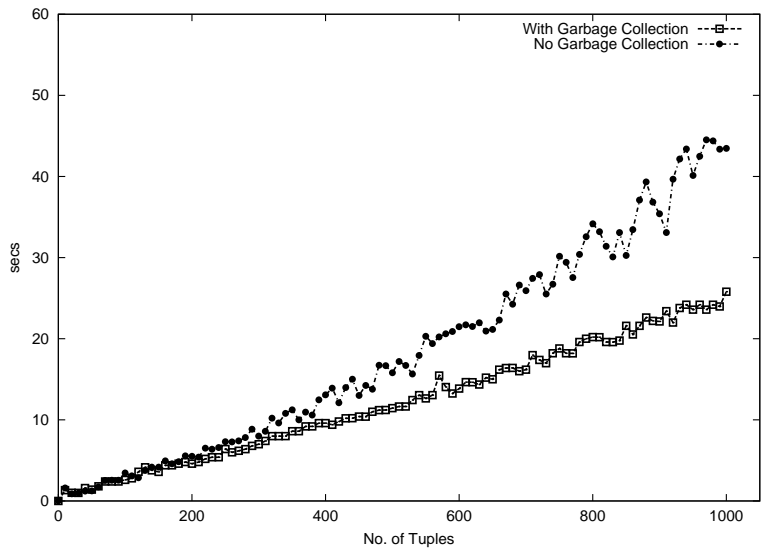

Figure 11: Garbage collector improving the system performance.

One should expect that in average case the overhead added by the the garbage collection scheme together with information gathering system is minimum (if existing). Several factors accounts for these results; first the garbage collector runs as a low priority thread which tries to use the processors idle time to run; second, the tuple monitoring only monitors tuples which contains handles and from experience it can be said that operations involving handles are not the commonest; finally, process registration and check-out only adds four extra messages (two for registration and two for check-out) between the given process and the kernel, and in the long run this adds practically nothing to the total execution time of a process.

\section{CONCLUSION}

This paper has proposed an efficient way of gathering information in open Linda-like systems which can be used as a source of information for optimizations. The paper has explored how garbage collection can be implemented using the distributed data structure containing the information gathered at run-time.

Due to the way the data structure is maintained a simpler garbage collection can be used as race-conditions are dealt with at the level of the data structure construction. The results have shown that not only the garbage collection does not add overhead to open Linda systems, the information gathering system does not have influence on its overall performance.

It has been argued that this solution is expected to work well under different assumptions. One case that is worth mentioning are models in which processes may not maintain references to tuple spaces as they can use lookup services to "discover" tuple spaces. It is important to realize that "something" must have a reference to the tuple space if it is still required. The solution proposed can easily adapt to consider lookup services as active processes with references to tuple spaces.

\section{ACKNOWLEDGMENTS}

I would like to thank Alan Wood from the University of York, UK, for his support and guidance during the development of the core work described in this paper. 


\section{REFERENCES}

[1] G. A. Agha. Actors: A Model of Concurrent Computation in Distributed Systems. The MIT Press, 1986.

[2] G. Berry and G. Boudol. The Chemical Abstract Machine. In ACM, editor, Proceedings of the seventeenth annual ACM symposium on Principles of programming languages, pages 81-94, New York, NY, USA, 1990. ACM Press.

[3] D. I. Bevan. Distributed Garbage Collection using Reference Counting. In W. J. Bakker, , L. Nijman, and P. C. Treleaven, editors, Proc. of Parallel Architectures and Languages Europe, pages 176-187. Springer-Verlag, June 1987. Lecture Notes in Computer Science, Vols. 258 and 259.

[4] L. Cardelli. Wide area computation. Technical report, Microsoft Research, UK, 1999.

[5] N. Carriero, D. Gelernter, and L. Zuck. Bauhaus Linda. In P. Ciancarini, O. Nierstrasz, and A. Yonezawa, editors, Object-Based Models and Languages for Concurrent Systems, volume 924 of Lecture Notes in Computer Science, pages 66-76. Springer-Verlag, Berlin, 1995.

[6] P. Ciancarini, A. Knoche, R. Tolksdorf, and F. Vitali. PageSpace: An Architecture to Coordinate Distributed Applications on the Web. In Proc. of the 5th Workshops on Enabling Technologies: Infrastructure for Collaborative Enterprises (WET ICE '96), pages 340-345. IEEE Computer Society Press, June 1996.

[7] G. E. Collins. A Method for Overlapping and Erasure of Lists. Communications of the ACM, 31(9):1128-1138, 1960.

[8] A. Douglas, A. Rowstron, and A. Wood. ISETL-LindA: Parallel Programming with Bags. Technical Report YCS-257, University of York, April 1995.

[9] A. Douglas, A. Wood, and A. Rowstron. LindA Implementation Revisited. In P. Nixon, editor, Proc. of the 18th World Occam and Transputer User Group, pages 125-138. IOS Press, April 1995.

[10] E. Freeman, S. Hupfer, and K. Arnold. JavaSpaces Principles, Patterns and Practice. Addison-Wesley, Reading, MA, USA, 1999. The Jini Technology Series.

[11] D. Gelernter. Generative Communication in LiNDA. ACM transactions in programming languages and systems, 1:80-112, 1985.

[12] D. Gelernter. Multiple Tuple Spaces in Linda. In Proc. of PARLE 89, pages 20-27. Springer-Verlag, 1989.

[13] D. Gelernter and N. Carriero. Coordination Languages and their Significance. Communications of the ACM, 35(2):96-107, February 1992.

[14] J. Hughes. A Distributed Garbage Collection Algorithm. In J.-P. Jouannaud, editor, Proc. of ACM Conference on Functional Programming Languages and Computer Architecture, pages 256-272, 1985. Lecture Notes in Computer Science, Vol. 201.

[15] S. C. Hupfer. Melinda: Linda with Multiple Tuple Space. Technical Report YALE/DCS/RR-766, Yale University, February 1990.

[16] IBM Corporation. T Spaces Programmer's Guide, 1998. Eletronic version only. http://www.almaden.ibm.com/cs/TSpaces/.
[17] R. Jones and R. Lins. Garbage Collection Algorithms for Automatic Dynamic Memory Management. Wiley, 1996.

[18] C. A. Knoblock and J. L. Ambite. Agents for Information Gathering. In J. Bradshaw, editor, Software Agents. AAAI/MIT Press, Menlo Park, CA, 1997.

[19] T. W. Malone and K. Crowston. What is Coordination Theory and How Can it Help Design Cooperative Work Systems? In Proceedings of the conference on Computer-supported cooperative work, CSCW' '90, pages 357-370, 1990.

[20] J. McCarthy. Recursive Functions of Symbolic Expressions and Their Computation by Machine. Communications of the ACM, 3(4):184-195, April 1960.

[21] R. Menezes and A. Wood. Coordination of Distributed I/O in Tuple Space Systems. In Proc. of 31st Hawaii International Conference on System Sciences, volume VII, pages 216-225, Big Island, Hawaii, USA, 1998. IEEE Computer Society.

[22] R. Menezes and A. Wood. Garbage Collection in Linda using Tuple Monitoring and Process Registration. In Proc. of the 10th International Conference on Parallel and Distributed Computing and Systems, pages 490-495, Las Vegas, Nevada, USA, 1998. Acta Press.

[23] R. Menezes and A. Wood. Ligia: A Java based Linda-like Run-time System with Garbage Collection of Tuple Spaces. Technical Report YCS-304, Department of Computer Science, University of York, September 1998.

[24] J. Piquer. Indirect Reference-Counting: A Distributed Garbage Collection Algorithm. In E. Odijik, M. Rem, and J.-C. Sayr, editors, Proc. of Parallel Architectures and Languages Europe, pages 150-165. Springer-Verlag, June 1991. Lecture Notes in Computer Science, Vols. 365 and 366.

[25] A. Rowstron and A. Wood. Bonita: A Set of Tuple Space Primitives for Distributed Coordination. In H. El-Rewini and Y. N. Patt, editors, Proc. of the 30th Hawaii International Conference on System Sciences, volume 1, pages 379-388. IEEE Computer Society Press, January 1997.

[26] Scientific Computing Associates Incorporated. Paradise - User's Guide and Reference Manual, 1994.

[27] Scientific Computing Associates Incorporated. LindA: An Introduction and Example. Available from Scientific Computing Associates Incorporated, One Century Tower, New Haven, CT 06510-7010, U.S.A, 1994.

[28] A. H. Sherman. C-Linda Reference Manual. Scientific Computing Associates, Inc., New Haven, Connecticut, 1990.

[29] Sun Microsystems, Inc. JavaSpace Specification, June 1997. Revision 0.4.

[30] Sun Microsystems, Inc. Jini Architecture Specification, January 1999. Revision 1.0.

[31] G. Sutcliffe and J. Pinakis. PROLOG-Linda: An Embedding of LINDA in muPROLOG. Technical report, The University of Western Australia, 1991. 